Pathologe 2008 · [Suppl 2] 29:319-327

DOI 10.1007/s00292-008-1038-7

Online publiziert: 10 . September

๑) Springer Medizin Verlag 2008

\author{
E. Noetzel ${ }^{1}$ J. Veeck ${ }^{1}$. F. Horn ${ }^{2}$ A. Hartmann ${ }^{3} \cdot$ R. Knüchel ${ }^{1}$ - E. Dahl ${ }^{1}$ \\ ${ }^{1}$ Arbeitsgruppe Molekulare Onkologie, Institut für \\ Pathologie, Universitätsklinikum Aachen \\ ${ }^{2}$ Klinik für Frauenheilkunde, Universität Regensburg \\ ${ }^{3}$ Institut für Pathologie, Universitätsklinikum Erlangen
}

\title{
Promotormethylierung von ID4
}

\section{Ein Marker für die Rezidivbildung des humanen Mammakarzinoms}

\begin{tabular}{|c|c|}
\hline Abkürzu & \\
\hline bHLH-Protein & Basisches Helix-Loop-Helix-Protein \\
\hline$B R C A$ & „breast cancer, early onset“ \\
\hline $\mathrm{CDH} 1$ & E-Cadherin \\
\hline$C T$ & "cycle treshhold" \\
\hline$D A C$ & 5-Aza-2'-Deoxycytidin \\
\hline DAPK & "death-associated protein kinase" \\
\hline$E R$ & Östrogenrezeptor \\
\hline ESR1 & Östrogenrezeptor 1 \\
\hline FC & „fold change" \\
\hline GAPDH & $\begin{array}{l}\text { Glyzerinaldehyd-3-Phosphat- } \\
\text { Dehydrogenase }\end{array}$ \\
\hline ID & $\begin{array}{l}\text { „inhibitor of DNA-binding"/ } \\
\text { "inhibitor of differentiation" }\end{array}$ \\
\hline IRS & Immunreaktiver Score \\
\hline HE-Färbung & Hämatoxylin-Eosin-Färbung \\
\hline HLH-Protein & Helix-Loop-Helix-Protein \\
\hline MGMT & $\begin{array}{l}\text { 0-6-Methylguanin-DNA-Methyl- } \\
\text { transferase }\end{array}$ \\
\hline $\begin{array}{l}\text { M-Reak- } \\
\text { tion }\end{array}$ & Primerreaktion mit methylierter DNA \\
\hline MSP & Methylierungsspezifische PCR \\
\hline OS & Gesamtüberleben \\
\hline$P A X$ & „paired box" \\
\hline$P C R$ & Polymerasekettenreaktion \\
\hline$P G R$ & Progesteronrezeptor \\
\hline$R B$ & Retinoblastom \\
\hline RFS & Rezidivfreies Überleben \\
\hline TIMP & „tissue inhibitor of metalloproteinase" \\
\hline TIMP3 & „TIMP metallopeptidase inhibitor $3^{\prime \prime}$ \\
\hline TNM & $\begin{array}{l}\text { Abkürzung für Tumor (Beschreibung } \\
\text { von Ausdehnung und Verhalten des } \\
\text { Primärtumors), ,„nodes“ (Lymphknoten, } \\
\text { Fehlen bzw. Vorhandensein von regio- } \\
\text { nalen Lymphknotenmetastasen) und } \\
\text { Metastasen (Fehlen bzw. Vorhanden- } \\
\text { sein von Fernmetastasen) }\end{array}$ \\
\hline TSA & Trichostatin A \\
\hline$T S P$ & Transkriptionsstartpunkt \\
\hline U-Reaktion & $\begin{array}{l}\text { Primerreaktion mit unmethylierter } \\
\text { DNA }\end{array}$ \\
\hline
\end{tabular}

Die ID-Protein-Familie besteht aus 4 Mitgliedern. ID 4 zeichnet sich wie alle IDProteine durch ein Helix-Loop-Helix-Motiv aus, mittels welchem es mit anderen basischen Helix-Loop-Helix-Proteinen dimerisieren kann. Unter ihren Bindungspartnern finden sich auch Nicht-bHLHProteine. Dazu gehören Transkriptionsfaktoren, wie das Retinoblastomaprotein oder die Gruppe der Pax-Proteine [1, 8]. Die Formation der ID-bHLH-Heterodimere führt zu einer dominant-negativen Kontrolle der Gentranskription und reguliert so die Aktivität von Transkriptionsfaktoren, welche für die Regulierung der Zellproliferation in der Entwicklung und der Differenzierung verschiedenster Zelltypen verantwortlich sind [33]. Es ist daher nicht überraschend, dass ein Zusammenhang zwischen der Deregulierung der ID-Gen-Expression und der humanen Krebsentstehung gezeigt werden konnte. In Karzinomen des Pankreas [15] und des Dickdarms $[15,29]$ ist die Genexpression von $I D_{1}, I D_{2}$ und $I D_{3}$ deutlich aufreguliert; folglich wurde diesen Genen primär ein onkogener Charakter zugeschrieben. ID4 hingegen scheint als Tumorsuppressor zu fungieren, da seine RNA-Expression in Prostatakarzinomen [1], humanen Leukämien [32], kolorektalen Karzinomen [25] und Adenokarzinomen des Magens [4] deutlich niedriger ist als in den entsprechenden Normalgeweben.

Aberrante epigenetische Modifikationen der DNA, z. B. Promotorhyper- methylierung, stellen zentrale Mechanismen der Genderegulierung in humanen Tumoren dar [14]. Die Hypermethylierung von CpG-reichen Regionen (CpGInseln) innerhalb von Genpromotoren ist eine wichtige Voraussetzung für die Inaktivierung von wichtigen Tumorsuppressorgenen, wie $p 16^{\mathrm{INKa}}, p 15^{\mathrm{INK} 4 \mathrm{~b}}, p_{14}{ }^{\mathrm{ARF}}$, $D A P K$ und MGMT [9]. Für das humane Mammakarzinom wurde bereits die epigenetische Inaktivierung durch eine Hypermethylierung für $B R C A 1,14-3-3 \sigma$, TIMP $3, E S R 1, P G R$ und $C D H_{1}$ beschrieben [10]. Die Promotorregion von $\mathrm{ID}_{4}$ enthält ebenfalls CpG-Inseln, welche in Adenokarzinomen des Magens als hypermethyliert gefunden wurden und mit dem Verlust der ID4-Gen-Expression assoziiert sind [4]. Verschiedene Studien postulierten eine mögliche Korrelation zwischen ID4-Promotor-Methylierung und Tumorinitiation bzw. -progression, z. B. in kolorektalen Karzinomen [25], humaner Leukämie [32] und Prostatakarzinom [1]. In normalen epithelialen Brustzellen wurde $\mathrm{ID}_{4}$ als konstitutiv exprimiert gefunden, die Expression geht jedoch bereits in präneoplastischen Läsionen verloren [7]. Eine andere Studie beschrieb ID4 als einen negativen Regulator des zentralen Brustkrebstumorsuppressorgens $B R C A 1$ [2, 24]. Darüber hinaus konnte ein Verlust der ID4-Expression in BRCA1/ER-positiven Brustkrebspatientinnen gezeigt werden, sodass angenommen wird, dass $\mathrm{ID}_{4}$ an der Regulie- 
rung der $E R$ - und BRCA1-Expression beteiligt ist [21]. Neben diesen Ergebnissen wird die Rolle von $\mathrm{ID}_{4}$ als putativem Tumorsuppressor kontrovers diskutiert und blieb bis jetzt ungeklärt. Im Widerspruch zum häufigen Verlust der ID4-Expression in humanen Tumorentitäten zeigte eine Studie die Aufregulierung von $\mathrm{ID}_{4}$ in der Brustdrüse der Ratte, verbunden mit einer gesteigerten Proliferation und Invasivität der Tumorzelllinien [22]. Eine andere Studie postulierte $I_{4}$ als Tumorsuppressor für die Subgruppe der T1-Mammakarzinome und zeigte, dass die aberrante Hypermethylierung des $\mathrm{ID}_{4}$-Gens mit einem erhöhten Risiko für Lymphknotenmetastasen assoziiert ist [26].

Die hier vorliegende Arbeit konzentriert sich auf die Klärung der Rolle der ID4-Promotor-Methylierung für die humane Brustkrebsentwicklung. Zu diesem Zweck analysierten wir eine klinisch-relevante Kohorte von kryokonservierten Gewebeproben von Brustkrebspatientinnen $(n=170)$. Dabei wurde besonderer Wert auf eine repräsentative Zusammenstellung bezüglich Tumorgröße und histologischem Grading gelegt. Mittels In-vitroDNA-Demethylierung [3] von humanen, malignen Brustkrebszelllinien wollten wir feststellen, inwiefern die ID4-PromotorMethylierung die ID4-mRNA-Expression beeinflussen kann. Ein weiteres Ziel war es, eine klare Beziehung zwischen $I_{4}$ Promotor-Methylierung, ID4-mRNAExpression und ID4-Protein-Expression in Mammakarzinomproben nachzuweisen. Eine derartige Korrelation wurde bis jetzt in keiner Studie gezeigt. Abschließend korrelierten wir die ID4-Promotor-Methylierung mit klinisch-pathologischen Parametern sowie der ID4-Expression von Brustkrebspatientinnen.

\section{Material und Methoden}

\section{Kryokonservierte Patientenproben}

Die Brustgewebeproben für die Methylierungs- und Expressionsanalysen stammen von Patientinnen $(n=170)$ mit primären Mammakarzinomen, die an den Universitätskliniken in Aachen, Jena, Regensburg und Düsseldorf (Deutschland) behandelt wurden. Alle Patientinnen gaben ihre Einverständniserklärung für die
Einbehaltung und Analyse von Gewebe für Forschungszwecke. Das Gewebematerial wurde sofort nach der Resektion in flüssigem Stickstoff eingefroren. Der Anteil an Tumorzellen in den Proben wurde mittels HE-Färbung bestimmt, nur solche Proben mit einem Anteil an Tumorzellen $>70 \%$ wurden für die weitere Analysen verwendet. Für Normalgewebe war ein Epithelanteil $>30 \%$ erforderlich.

\section{Zelllinien}

Die verwendeten humanen Brustzelllinien BT20, MDA-MB231, MCF7 und T47D wurden von der American Type Culture Collection (ATCC, Rockville, MD) bezogen und den Herstellerangaben entsprechend kultiviert.

\section{Nukleinsäureextraktionen}

DNA-Präparationen aus Zelllinien und humanem Gewebe wurde unter Verwendung des QiAmp ${ }^{\oplus}$-DNA-Kit (Qiagen, Hilden) und den vom Hersteller empfohlenen Bedingungen gewonnen. RNA-Präparationen wurden entsprechend dem TRIzol ${ }^{\oplus}$-Verfahren nach Herstellerangaben durchgeführt (Invitrogen, Carlsbad, CA).

\section{Reverse Transkription}

$1 \mu \mathrm{g}$ Gesamt-RNA wurde mit dem Promega-Reverse-Transcription-System (Promega, Madison, WI) nach Herstellerangaben in cDNA umgeschrieben.

\section{Semiquantitative Echtzeit-PCR}

Die Echtzeit-PCR wurde mit dem LightCycler ${ }^{\circledast}$-System (Roche Diagnostics, Mannheim) unter Verwendung Intron überspannender Primer nach Herstellerangaben wie beschrieben durchgeführt und mittels komparativer CT-Methode im Vergleich zur GAPDH-Expression ausgewertet $[11,17,27]$.

\section{Bisulfitmodifikation und methylierungsspezifische PCR}

Sie wurden wie in der Literatur beschrieben durchgeführt $[5,12,17,27]$.

\section{In-vitro-Demethylierung von DNA}

Tumorzelllinien wurden in einer Dichtevon $3 \times 10^{4}$ Zellen $/ \mathrm{cm}^{2}$ am Tag o in einer 6-LochSchale ausgesät. Die In-vitro-Demethylierung wurde mit dem Agens 5-Aza-2'Deoxycytidin (Sigma-Aldrich, Deisenheim) und dem Histondeazetylaseinhibitor Trichostatin A (Sigma-Aldrich, Deisenheim) wie beschrieben durchgeführt [17].

\section{Immunhistochemie}

Für die ID4-Protein-Färbung wurden $3 \mu \mathrm{m}$ dicke, formalinfixierte, paraffineingebettete Gewebeschnitte für $30 \mathrm{~min}$ bei $72^{\circ} \mathrm{C}$ getrocknet, in Xylen entparaffiniert und in einer absteigenden Alkoholreihe rehydriert. Anschließend wurden die Schnitte für $35 \mathrm{~min}$ in Tris-EDTA-Puffer ( $\mathrm{pH}$ 9,o) zwecks Antigendemaskierung bei $95^{\circ} \mathrm{C}$ inkubiert. Es wurde ein polyklonaler ID4-Antikörper (Verdünnung 1:150; Inkubation für $90 \mathrm{~min}$; Kaninchen-antiHuman; Sc291, Santa Cruz, CA) verwendet. Der immunhistochemische Nachweis erfolgte unter Verwendung des ChemMate $^{\mathrm{Tm}}$ EnVision $^{\mathrm{Tm}}$ Kit (DAKO, Hamburg). Die Schnitte wurden mit Mayer's Hematoxylin gegengefärbt und mit Entellan Einbettmedium (EMS Diatome, Fort Washington, PA) fixiert. Normal- und Tumorgewebe des Dickdarms dienten als Positivkontrollen für die $\mathrm{ID}_{4}$-ProteinFärbung [25]. Die Färbungsintensität wurde von erfahrenen Pathologen nach dem System von Remmele u. Stegner [20] bewertet.

\section{Statistische Methoden}

Die statistische Analyse wurde mit der Software SPSS 14.0 durchgeführt (SPSS Software $\mathrm{GmbH}$, München). Eine statistische Signifikanz wurde ab einem $\mathrm{p}<0,05$ angenommen. Ein zweiseitiger KruskalWallis-Test wurde verwendet, um die Expressionsunterschiede zwischen unmethylierten Tumoren, methylierten Tumoren und Normalgeweben zu analysieren. Assoziationen zwischen klinisch-pathologischen Faktoren und molekularen $\mathrm{Pa}$ rametern wurden mittels Fisher's-ExaktTest untersucht. Das rezidivfreie Überleben und das Gesamtüberlebensintervall 
in Relation zu klinisch-pathologischen und molekularen Parametern wurden mittels univariatem Log-rank-Test nach der Methode von Kaplan-Meier sowie mittels multivariater Cox-Regression berechnet.

\section{Ergebnisse}

\section{IID4-Methylierung und}

ID4-Reexpression durch In-vitro-Demethylierung in humanen Brustzelllinien

Initial wurde eine methylierungsspezifische PCR für die Promotoranalyse des ID4-Gens etabliert. Hierfür wurden MSPPrimer verwendet, welche komplementär zu einer Sequenz innerhalb der zentralen CpG-Insel $(-615$ bis +139$)$ nahe des TSP des $I_{4}$-Promoters sind ( $\bullet$ Abb. 1a) und einen Sequenzabschnitt, der etwa 30 bp stromaufwärts des TSP beginnt, amplifizieren. Das PCR-Amplikon des unmethylierten ID4-Promotors war $161 \mathrm{bp}$ groß, das des methylierten $\mathrm{ID}_{4}$-Promotors $157 \mathrm{bp}$. Die für die Reaktion mit unmethylierter DNA verwendeten Primer sind komplementär zu den Basen -194 bis -166 und -60 bis -33 . Entsprechend binden die für die Reaktion mit methylierter DNA eingesetzten Primer die Basen -192 bis -166 und -60 bis -35 (alle Positionen relativ zum TSP).

Um eine direkte Assoziation zwischen der ID4-Promotor-Methylierung und der ID4-Gen-Expression nachzuweisen, wurde eine In-vitro-Demethylierungsanalyse an 4 humanen, malignen Brustkrebszelllinien durchgeführt (MDA-MB231, BT2O, MCF7 und $\mathrm{T}_{47} \mathrm{D}$ ). Dazu wurden diese Zelllinien mit dem Demethylierunsagens DAC (72 h) und dem Histondeazetylaseinhibitor TSA $(24 \mathrm{~h})$ inkubiert. Die ID4Expression wurde anschließend mittels semiquantitativer Echtzeit-PCR bestimmt (- Abb. 1b). Alle methylierten Zelllinien wiesen nach der Behandlung eine gesteigerte ID4-Expression auf, T47D 119-fach, MCF7 38-fach und BT20 19-fach. Die unmethylierte Zelllinie MDA-MB231 dagegen zeigte nur eine marginale Alteration in ihrer ID4-mRNA-Expression.

Pathologe 2008 • [Suppl 2] 29:319-327 DOI 10.1007/s00292-008-1038-7

(c) Springer Medizin Verlag 2008

\section{E. Noetzel · J. Veeck · F. Horn · A. Hartmann · R. Knüchel · E. Dahl \\ Promotormethylierung von ID4. Ein Marker für die Rezidivbildung des humanen Mammakarzinoms}

\section{Zusammenfassung}

In der vorliegenden Studie sollte die Rolle des Transkriptionsfaktors „inhibitor of DNA binding 4" (ID4) in der humanen Brustkrebsentwicklung detaillierter untersucht werden, insbesondere die Bedeutung von aberranter ID4-Promotor-Methylierung für den Krankheitsprozess. ID4-Gen-Methylierung zeigte sich in 68,9\% (117/170) der untersuchten Mammakarzinompräparate und korrelierte hochsignifikant mit dem ID4-mRNA-Expressionsverlust $(p<0,001)$. Erstmalig konnte eine direkte Korrelation zwischen der ID4-Promotor-Methylierung, einer ID4-mRNA-Herabregulierung und dem Verlust der ID4-Protein-Expression nachgewiesen werden. Interessanterweise erwies sich die ID4-PromotorMethylierung als Marker für ein ungünstiges rezidivfreies Überleben $(p=0,036)$ und zeigte ein erhöhtes Risiko für Lymphknotenmetastasen $(p<0,030)$ an. Unsere Ergebnisse deuten darauf hin, dass ID4 ein neues Tumorsuppressorgen des humanen Brustgewebes darstellen könnte. Es wird im Zuge der Tumorentstehung frequent epigenetisch abgeschaltet, die entsprechenden Mammakarzinome haben möglicherweise ein erhöhtes Rezidivrisiko. ID4-Promotor-Methylierung könnte daher als prognostischer Biomarker für das humane Mammakarzinom genutzt werden.

Schlüsselwörter ID4-Promotor-Methylierung · Brustkrebs . Rezidivfreies Überleben · Lymphknotenmetastasen · Tumorsuppressorgen

\section{Promoter methylation of ID4. A marker for recurrence- free survival in human breast cancer}

\section{Abstract}

The aim of this study was to unravel the role of the transcription factor inhibitor of DNA binding 4 (ID4) in human breast carcinogenesis in more detail, especially the impact of ID4 promoter methylation on disease progression. Demethylating treatment of breast cancer cell lines was associated with ID4 reexpression. ID4 promoter methylation was frequently observed in primary breast cancer samples $(68.9 \%, 117 / 170)$. We found a very tight correlation $(p<0.001)$ between ID4 promoter methylation and loss of ID4 mRNA expression in these specimens. For breast tissue as the first tumour entity analyzed in detail, we could show a direct correlation between ID4 promoter methylation and loss of ID4 expression on both the mRNA and pro- tein level.

Interestingly, ID4 promoter methylation was a factor for unfavourable recurrence-free survival $(p=0.036)$ and increased the patient's risk for lymph node metastases ( $p=0.030$ ). Our data suggest that ID4 is a potential tumour suppressor gene in human breast tissues that undergoes epigenetic silencing during carcinogenesis, leading to an increased risk for tumour relapse. Thus, ID4 methylation status could serve as a prognostic biomarker in human breast cancer.

Keywords

ID4 promotor methylation - Breast cancer . Recurrence free survival · Lymph node metastases - Tumour suppressor gene 


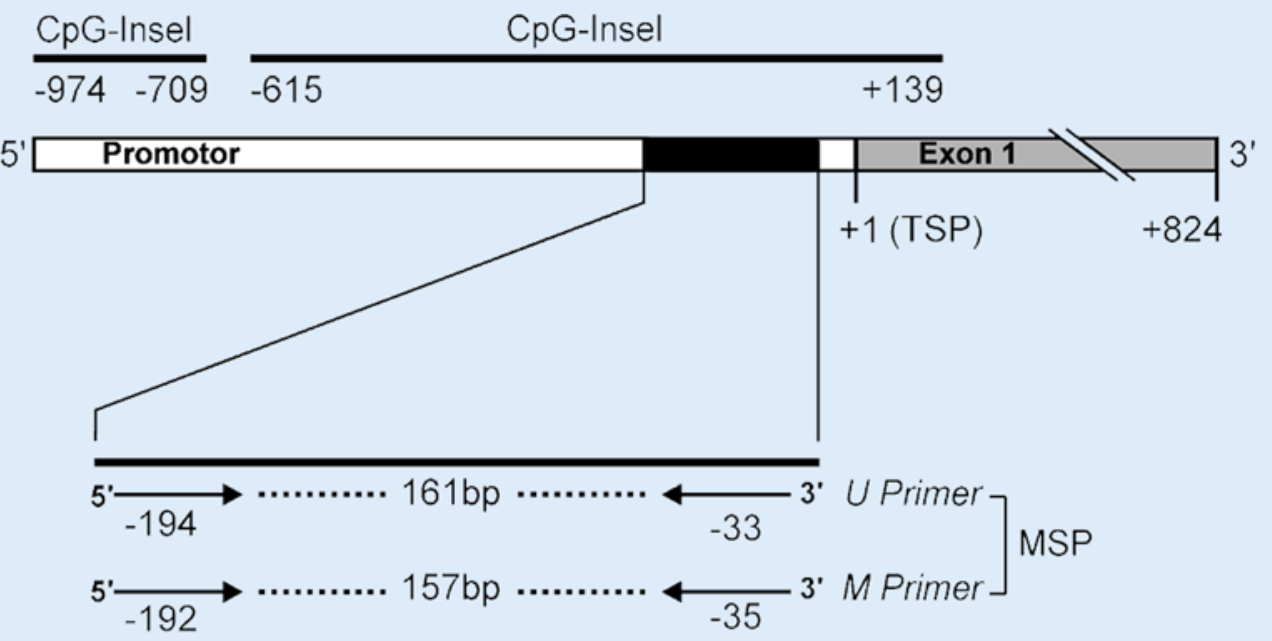

a

\begin{tabular}{|c|c|c|c|c|c|c|c|c|}
\hline & \multicolumn{2}{|c|}{ MDA-MB231 } & \multicolumn{2}{|c|}{ BT20 } & \multicolumn{2}{|c|}{ MCF7 } & \multicolumn{2}{|c|}{ T47D } \\
\hline & Kontrolle & $\begin{array}{l}\text { DAC } \\
\text { TSA }\end{array}$ & Kontrolle & $\begin{array}{l}\text { DAC } \\
\text { TSA } \\
\end{array}$ & Kontrolle & $\begin{array}{l}\text { DAC } \\
\text { TSA } \\
\end{array}$ & Kontrolle & $\begin{array}{l}\text { DAC } \\
\text { TSA }\end{array}$ \\
\hline$M C D$ & $\mathbf{U} \mathbf{M}$ & $U M$ & U M & U M & $\mathbf{U} \quad \mathbf{M}$ & $U \mathrm{M}$ & $\mathbf{U} \mathbf{M}$ & $U M$ \\
\hline $\begin{array}{lc}\text { Echt } & \text { Zeit } \\
\text { Zeit } & \text { FC }\end{array}$ & 1 & 1.8 & 1 & 19.4 & 1 & 37.5 & 1 & 119.3 \\
\hline
\end{tabular}

b

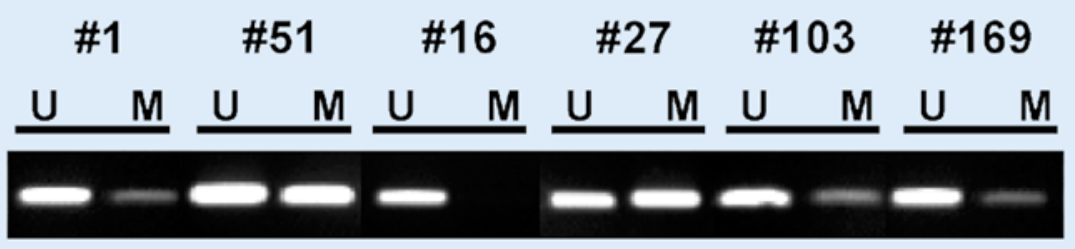

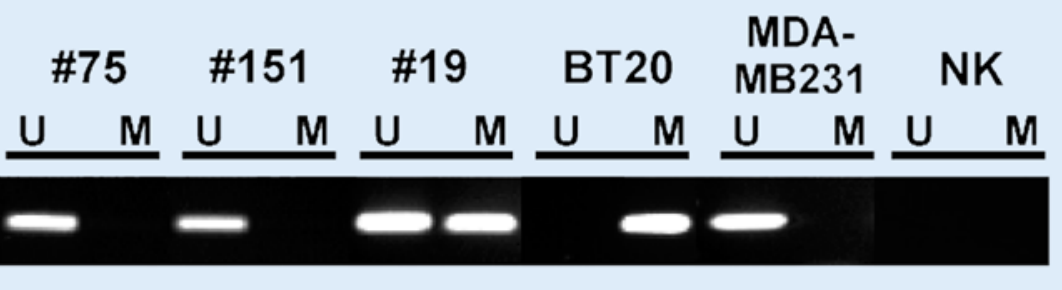

\section{ID4-Promotor-Methylierung in primären, humanen \\ Mammakarzinomen}

Wir konnten bereits demonstrieren, dass die ID 4 -mRNA-Expression in 78\% (39/50) aller humanen, primären Mammakarzinomen signifikant herabreguliert ist [25]. Umetani et al. [26, 25] implizierten in einer Studie, dass die Promotorhypermethylierung ein effektiver Mechanismus der ID4Inaktivierung im humanen Brustkrebs darstelle, wobei sich ihre Untersuchungen auf die Subgruppe von T1-Mammakarzinomen beschränkten [19]. Ein Ziel dieser Studie war es daher, die exakte Methylierungsfrequenz des $I_{4}$-Promoters beim humanen Mammakarzinom anhand eines klinisch relevanten Spektrums von Brustkrebspatientinnen zu bestimmen. Hierzu analysierten wir genomische DNA von 170 Patientinnen mit primären Mammakarzinomen mittels MSP-Technologie. Repräsentative Ergebnisse von 9 Patien-
Abb. $1<$ a lllustration der ID4-Gen-Promotor-Region und MSP-Primer-Topologie, Lokalisation von $2 \mathrm{CpG}$ Inseln innerhalb der ID4Promotor-Region stromaufwärts des TSP, schwarzer Balken Amplikon, U-Primer Bindung an unmethylierte, M-Primer Bindung an methylierte DNA, b In-vitro-Demethylierungsanalyse des ID4-Promotors bei 4 humanen Brustkrebszelllinien (MDA-MB231, BT20, MCF7, T47D), ID4-Methylierungsstatus und $F C$ der ID4-mRNA-Reexpression, DAC 5-Aza-2'-Deoxycytidin, TSA Trichostatin A, c ID4Promotor-Methylierungsanalyse primärer, humaner Mammakarzinome mittels MSP-Technologie, BT20, MDA-MB231 Positivkontrollen, NK Negativkontrolle. (Mit Genehmigung aus [17]), U unmethylierte, $M$ methylierte DNA, Erläuterung s. Text 
kontrolle der MSP. Insgesamt konnte eine ID4-Promotor-Methylierung in 68,9\% (117/170) der Patientinnen nachgewiesen werden. Dementsprechend zeigten 31,1\% (53/170) keine ID4-Promotor-Methylierung.

Zusätzlich wurden 13 Normalgewebe getestet, von denen keine einzige Probe eine ID4-Methylierung zeigte. Somit konnten wir zeigen, dass es sich bei der aberranten Hypermethylierung des ID4-Promotors um einen tumorspezifischen $\mathrm{Me}$ chanismus handelt.

\section{Korrelation zwischen ID4- Promotor-Methylierung und ID4-Expression im humanen Mammakarzinom}

Unser nächstes Ziel war es, zu analysieren, inwiefern ID4-Promotor-Methylierung zwangsläufig auch zu einer Inaktivierung des $I_{4}$-Promtors führt. $\mathrm{Zu}$ diesem Zweck wurde für einen Teil der Kohorte $(n=46)$, für die der ID4-Promotor-Methylierungsstatus bestimmt wurde, auch die ID4-mRNA-Expressionsstärke ermittelt (• Abb. 2a). Im direkten Vergleich zu einem Standard aus 12 gepoolten Brustnormalgeweben $(\mathrm{N})$ erwies sich der $\mathrm{ID}_{4}$ mRNA-Verlust in den unmethylierten Tumoren $(\mathrm{T})$ als marginal $(\mathrm{FC}=\mathrm{N} / \mathrm{T}=1,3)$. Im Gegensatz dazu wiesen $I_{4}$-methylierte Mammakarzinome einen hochsignifikanten Verlust ( $\mathrm{p}<\mathrm{0}, \mathrm{oo1})$ der ID4-Expression mit einem $\mathrm{FC}=12,3$ auf. Diese Daten zeigen eindeutig, dass die ID4-Promotor-Methylierung mit der $I_{4}-$ Gen-Inaktivierung assoziiert ist. Die Analyse der ID4-mRNA-Expression in Mammakarzinomen und entsprechenden Mammanormalgeweben bestätigte einen Verlust der ID4-mRNA in $82 \%$ der Tumoren (bei einem $\mathrm{FC} \geq 2$ ).

Um nachzuweisen, dass die ID4-Promotor-Methylierung ebenfalls zu einem Verlust des ID4-Proteins führt, wurden parallel der ID4-Methylierungs-Status, die ID4-mRNA-Expression und die ID4Protein-Expression bei 3 Patientinnen bestimmt, von denen jeweils Tumor- (T) und korrespondierendes Brustnormalgewebe $(\mathrm{N})$ verfügbar waren (• Abb. 3). Brustkrebspatientinnen mit einem unmethylierten $\mathrm{ID}_{4}$-Promotor wiesen nur einen marginalen Unterschied ( $F C=1,1$, über den

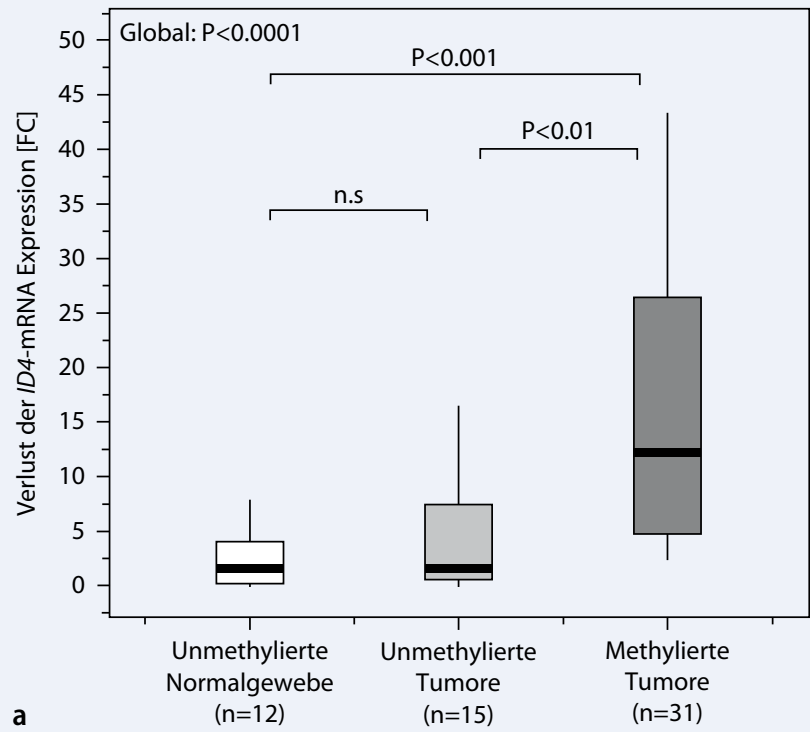

Abb. 2 a Box-PlotDarstellung des Verlusts der ID4-Expression in Abhängigkeit vom Methylierungsstatus des ID4-Promotors bei Brustkrebspatientinnen, $Y$-Achse Faktor der ID4-mRNA-Herabregulierung in Brustkrebspatientinnen $(\mathrm{n}=46)$ relativ zum Normalgewebestandard (FC), horizontale Linien Mediane der Gruppen; Kästen 25bis 75\%-Quartile; vertikale Linien Spannweite, Minimum und Maximum, b Kaplan-Meier-Analyse des rezidivfreien Überlebens (RFS) von Brustkrebspatientinnen in Abhängigkeit der ID4-PromotorMethylierung, Zeitverlauf (Monate) und RFS von 115 Brustkrebspatientinnen mit positiver (untere Kurve) und negativer (obere Kurve) ID4-Promotor-Methylierung, Details s. Text. (Mit Genehmigung aus [17])

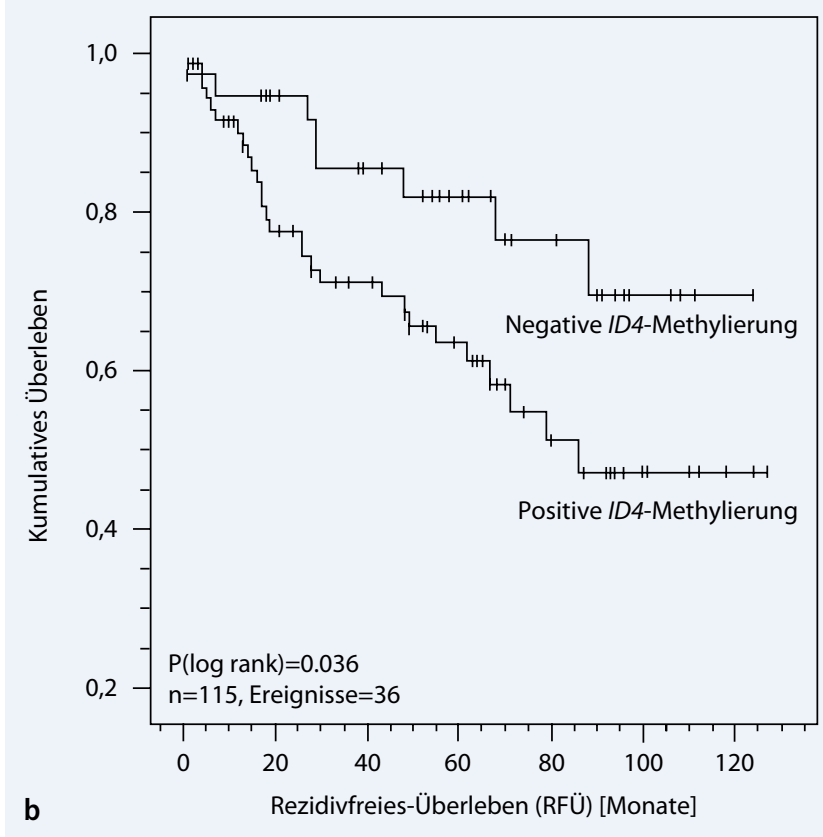

Quotienten der Expression von N und T berechnet) in ihrer ID4-mRNA Expression verglichen mit dem entsprechenden Normalgewebe auf. Dementsprechend zeigte sich auf der Proteinebene ebenfalls kein Unterschied der ID4-Expression zwischen Normal- und Tumorgewebe. Mammakarzinompatientinnen mit einem methylierten $I_{4}$-Promotor hingegen wiesen einen starken Verlust der ID4-mRNA auf (16-fach bis 71-fach), verglichen mit den entsprechenden Normalgeweben. Diese methylierten Tumoren zeigten ebenfalls einen fast vollständigen Verlust der $\mathrm{ID}_{4}$ Protein-Expression (• Abb. 3b,c).

\section{Klinische Relevanz der ID4- Promotor-Methylierung für das humane Mammakarzinom}

Korrelationsanalysen der ID4-PromotorMethylierung mit klinisch-pathologischen Parametern von Mammakarzinompatientinnen wurden mit dem Fischer's-ExaktTest durchgeführt (• Tab. 1). Ein hypermethylierter ID4-Promotor war hierbei signifikant mit einem positiven Lymphknotenstatus $(\mathrm{p}=\mathrm{0}, 03 \mathrm{O})$ und einem Verlust der ID4-mRNA-Expression ( $\mathrm{p}<0,001)$ assoziiert. Es wurden keine Korrelationen zwischen rezidivfreiem Überleben und Gesamtüberleben mit dem Alter, der Tumorgröße, dem histologischen Grading/Typ 
Beste Forschungsbeiträge: Gynäkopathologie

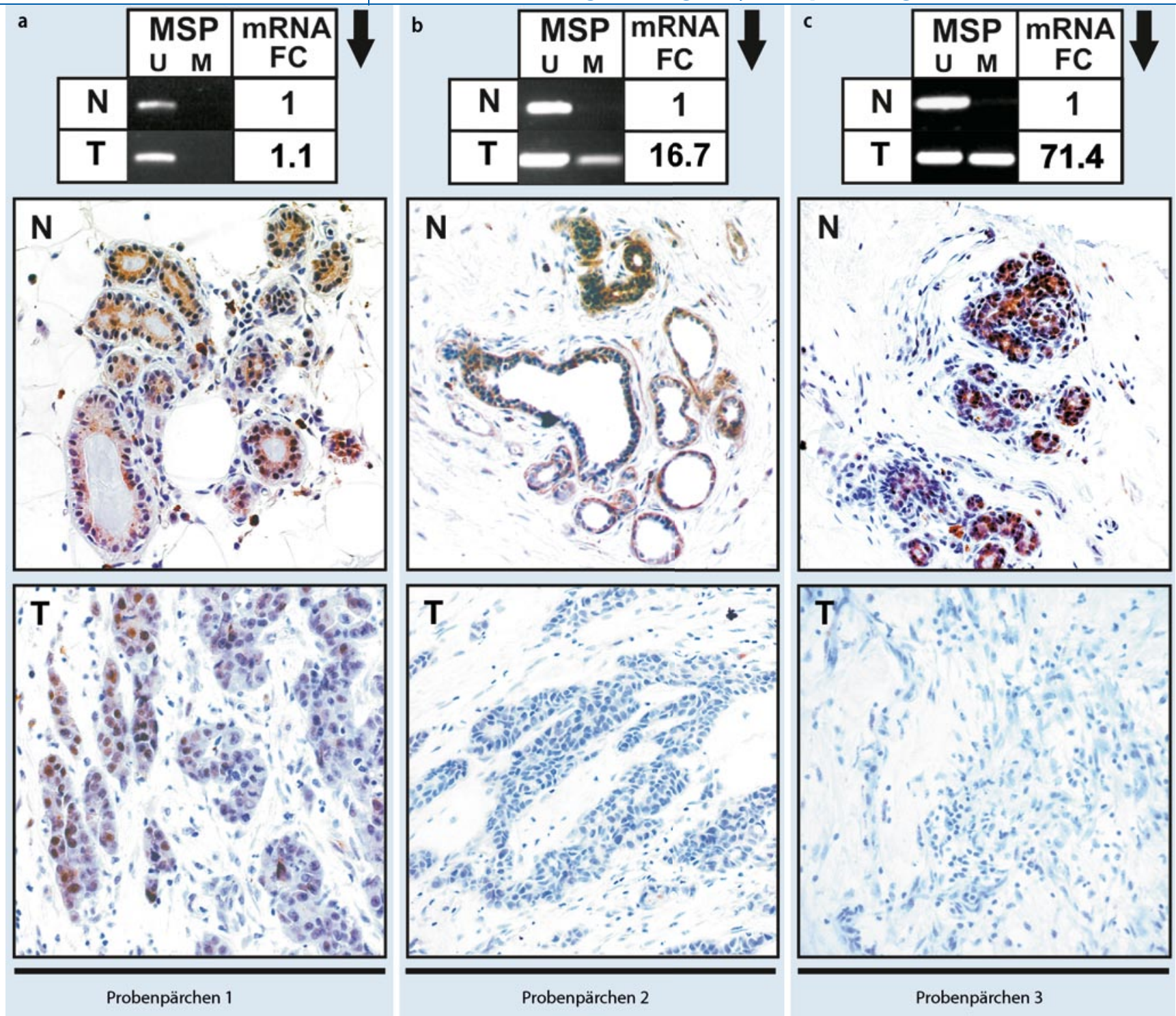

Abb. $3 \Delta$ Kohärenz der ID4-Promotor-Methlyierung und der ID4-Expression in 3 repräsentativen Proben von primären Mammakarzinomen ( $T$ ) und ihren korrespondierenden Normalgeweben (N), MSP: ID4-Methylierungsstatus, mRNA-FC: ID4-mRNAExpression, Gewebeproben: immunohistochemischen Färbung mit einem ID4-Antikörper, Vergr. 400:1, a unmethylierter Tumor, im direktem Vergleich zu korrespondierendem Normalgewebe, nur marginal veränderte ID4-mRNA- und ID4-ProteinExpression. b, c methylierte Tumoren, deutlicher Verlust der ID4-mRNA- und ID4-Protein-Expression im Vergleich zu entsprechendem Normalgewebe, Details im Text. (Mit Genehmigung aus [17])

bzw. dem Östrogen-/Progesteronrezeptorstatus gefunden. Der direkte Vergleich des RFS, des OS und des ID4-Methylierungsstatus ist in $\bullet$ Tab. 2 zusammengefasst. Wir fanden ein erhöhtes Risiko für die Rezidivbildung bei Mammakarzinompatientinnen mit positiver $I_{4}$-Promotor-Methylierung $(\mathrm{p}=\mathrm{O}, \mathrm{O} 36)$, verglichen mit $\mathrm{Pa}-$ tientinnen ohne $I D_{4}$-Methylierung: Erstere hatten eine geschätzte mittlere RFS-Zeit von 80 Monaten (95\%-Konfidenzintervall: 67-93 Monate) verglichen mit 101 Monaten (95\%-Konfidenzintervall: 87-115 Mo- nate) bei Patientinnen ohne ID4-Methylierung (• Abb. 2b, [17]).

Die statistische Abschätzung erfolgte mittels Kaplan-Meier-Methode. Die ID4Promotor-Methylierung war hierbei mit einer niedrigeren 10-Jahres-RFS-Rate von $47 \%$ assoziiert, während Patientinnen ohne ID4-Promotor-Methylierung eine 10Jahres-RFS von $71 \%$ aufwiesen. Der $I D_{4}$ Promotor-Methylierungsstatus erwies sich in einem multivariaten Cox-Regressionsmodell inklusive der Faktoren, welche das RFS beeinflussen könnten, als nicht signifikant (Daten nicht gezeigt).
Ein Grund hierfür könnte die starke Assoziation zwischen der ID4-Methylierung und dem Risiko für Lymphknotenmetastasen sein.

\section{Diskussion}

Es ist bekannt, dass der HLH-Transkriptionsfaktor $\mathrm{ID}_{4}$ funktionell mit fundamentalen Prozessen von Zellen wie Differenzierung, Proliferation, Apoptose und Angiogenese verknüpft ist [33] und mit Zellzyklusfaktoren wie dem RB1-Protein oder PAX-Proteinen interagiert [31]. Aus die- 
sem Grund ist es nicht verwunderlich, dass eine Deregulierung von ID-Proteinen in verschiedenen Tumorentitäten gefunden wurde [18]. Die epigenetische Inaktivierung des $I D_{4}$-Gens durch aberrante Promotorhypermethylierung konnte bereits für diverse humane Tumorentitäten gezeigt werden, wie für Karzinome des Magens [4] und des Dickdarms [25]. Für das humane Mammakarzinom wurde eine solche epigenetische Regulierung der ID4-Expression in 67\% (16/24) der nodal-positiven Tumoren demonstriert, wobei sich die Analyse auf eine Subgruppe von T1-Tumoren beschränkte [26]. Aus diesem Grund sollte in der vorliegenden Arbeit die Auswirkung einer aberranten ID4-Promotor-Methylierung anhand einer klinisch-relevanten Kohorte von Patientinnen mit primären humanen Mammakarzinomen sowie in verschiedenen humanen Brustkrebszelllinien analysiert werden.

Wir konnten durch ein In-vitro-Demethylierungsexperiment in humanen Brustkrebszelllinien zeigen, dass die $I D_{4}$ Expression nach der ID4-Promotor-Demethylierung wiederhergestellt wird. Daraus lässt sich schlussfolgern, dass die ID4-Promotor-Methylierung ein zentraler Mechanismus für die Abschaltung des ID4-Gens in humanen Mammakarzinomen ist. Dieser Befund ist eine elementare Voraussetzung für eine mögliche tumorsuppressive Funktion des unmethylierten ID4-Gens in der humanen Brust. Bis jetzt konnte eine epigenetische Inaktivierung von $I D_{4}$ nur für Karzinome des Magens [4] und des Dickdarms [25] gezeigt werden. In dieser Arbeit konnten wir belegen, dass auch ein hoher Prozentsatz von Mammakarzinomen (69\%) eine aberrante $I_{4}$-Promotor-Methylierung aufweist. Ferner konnten wir zeigen, dass es zu einem hochsignifikanten Verlust der ID4-mRNA durch eine ID4-Promotor-Methylierung in $83 \%$ aller Brustkrebspatientinnen kommt. Diese Frequenz ist vergleichbar zu der bereits beschriebenen der $I_{4}$-mRNA-Herabregulierung in $78 \%$ der Fälle auf einer cDNADot-Blot-Analyse [6]. Allerdings stehen unsere Ergebnisse im Widerspruch zu einer bereits berichteten ID4-mRNA-Heraufregulierung in Brustkrebszellen der Ratte [22].

\begin{tabular}{|c|c|c|c|c|c|}
\hline \multirow[t]{2}{*}{ Variable } & \multicolumn{5}{|l|}{ ID4-Methylierung } \\
\hline & & Anzahl (n) ${ }^{\mathrm{a}}$ & Positiv & Negativ & $\mathrm{p}$-Wert ${ }^{\mathrm{d}}$ \\
\hline Total & & 170 & 117 & 53 & \\
\hline \multicolumn{6}{|c|}{ Klinisch-pathologische Parameter } \\
\hline \multirow[t]{2}{*}{ Alter bei Diagnose } & $<50$ Jahre & 51 & 34 & 17 & \multirow[t]{2}{*}{0,821} \\
\hline & $\geq 50$ Jahre & 111 & 76 & 35 & \\
\hline \multirow[t]{2}{*}{ Tumorgröße $^{b}$} & pT1 & 62 & 24 & 44 & \multirow[t]{2}{*}{0,247} \\
\hline & pT2-4 & 91 & 27 & 7 & \\
\hline \multirow[t]{2}{*}{ Lymphknotenstatus $^{b}$} & pNO & 74 & 43 & 31 & \multirow[t]{2}{*}{0,030} \\
\hline & $\mathrm{pN} 1-3$ & 62 & 47 & 15 & \\
\hline \multirow{3}{*}{$\begin{array}{l}\text { Histologisches } \\
\text { Grading }\end{array}$} & G1 & 14 & 10 & 4 & \multirow[t]{3}{*}{0,944} \\
\hline & $\mathrm{G} 2$ & 79 & 52 & 27 & \\
\hline & G3 & 61 & 42 & 19 & \\
\hline \multirow{3}{*}{$\begin{array}{l}\text { Histologischer } \\
\text { Tumortyp }\end{array}$} & Invasiv duktal & 129 & 87 & 42 & \multirow[t]{3}{*}{0,940} \\
\hline & Invasiv lobulär & 18 & 12 & 6 & \\
\hline & Andere & 12 & 8 & 4 & \\
\hline \multirow{2}{*}{$\begin{array}{l}\text { Östrogen- } \\
\text { rezeptorstatus }\end{array}$} & Negativ $\left(\operatorname{IRS}^{c}=0-2\right)$ & 52 & 35 & 17 & \multirow[t]{2}{*}{0,900} \\
\hline & Positiv (IRS=3-12) & 101 & 69 & 32 & \\
\hline \multirow{2}{*}{$\begin{array}{l}\text { Progesteron- } \\
\text { rezeptorstatus }\end{array}$} & Negativ (IRS $=0-2)$ & 57 & 39 & 18 & \multirow[t]{2}{*}{0,920} \\
\hline & Positiv (IRS=3-12) & 96 & 65 & 31 & \\
\hline \multirow{2}{*}{$\begin{array}{l}\text { ID4-mRNA- } \\
\text { Expression }\end{array}$} & $\mathrm{FC}<2$ & 8 & 0 & 8 & \multirow[t]{2}{*}{$<0,001$} \\
\hline & $\mathrm{FC} \geq 2$ & 38 & 31 & 7 & \\
\hline \multicolumn{6}{|c|}{$\begin{array}{l}\text { aPatientinnen mit primären, unilateralen, invasiven Mammakarzinomen, kursiv signifikante p-Werte } \\
\text { bEntsprechend der TMN-Klassifikation von Sobin u. Wittekind [23] } \\
\text { 'IRS Immunreaktiver Score nach [20] } \\
\text { dFischer's-Exakt-Test }\end{array}$} \\
\hline
\end{tabular}

Unsere statistischen Analysen verdeutlichten, dass es sich bei der ID4-PromotorMethylierung um einen neuen, negativprognostischen Faktor handeln könnte. Brustkrebspatientinnen mit einem methylierten $I D_{4}$-Promotor zeigten ein verkürztes RFS im direkten Vergleich zu Patientinnen ohne ID4-Promotor-Methylierung. Dieser Befund stützt wiederum unsere Hypothese, dass ein funktionelles ID4-Gen eine tumorsuppressive Funktion im humanen Brustgewebe ausübt. Somit könnte $I D_{4}$ eine gegensätzliche zelluläre Funktion im Bezug zu $I D_{1}$ und $I D_{2}$ besitzen, denen eine onkogene Rolle in der humanen Brust zugesprochen wird $[13,16,19]$. Die statistische Auswertung unserer Kohorte von Brustkrebspatientinnen $(\mathrm{n}=170)$ zeigte eine enge Korrelation zwischen ID4-Promotor-Methylierung und dem Risiko für Lymphknotenmetastasen. Dieser Zusammenhang wurde ebenfalls für eine kleine Subgruppe von T1-Tumoren in der Studie von Umetani et al. [26] gefunden und weist auf eine metastasierungssuppressive Funktion von $I D_{4}$ hin. Darüber hinaus wurden kei- ne signifikanten Korrelationen zwischen der ID4-Promotor-Methylierung und anderen klinisch-pathologischen Parametern festgestellt.

Unserem Wissensstand nach ist die vorliegende Arbeit die erste Studie, welche einen deutlichen Verlust der ID4-ProteinExpression und der ID4-mRNA-Expression in Assoziation mit der ID4-Promotor-Hypermethylierung für das humane Mammakarzinom nachweisen konnte. Der Nachweis des Proteinverlusts, welcher letztendlich die Aktivität von Zielgenen moduliert, stellt ein wichtiges Ergebnis für die Validierung von $\mathrm{ID}_{4}$ als einem neuen Tumorsuppressorgen der humanen Mamma dar. Zuvor konnte zwar bereits ein ID4-Protein-Verlust in Mamma- [28] und kolorektalen Karzinomen [25] gezeigt werden, diese Studien wiesen aber nicht den Zusammenhang des Proteinverlusts zur Methylierung des ID4-Promotors nach. 


\begin{tabular}{|c|c|c|c|c|c|c|c|}
\hline \multirow[t]{2}{*}{ Variable } & & \multicolumn{3}{|c|}{ Rezidivfreies Überleben } & \multicolumn{3}{|c|}{ Gesamtüberleben } \\
\hline & & $\begin{array}{l}\text { Anzahl } \\
(n)^{a}\end{array}$ & Ereignisse & $p$-Wert ${ }^{d}$ & $\begin{array}{l}\text { Anzahl } \\
(n)^{a}\end{array}$ & Ereignisse & p-Wert ${ }^{d}$ \\
\hline \multicolumn{8}{|c|}{ Klinisch-pathologische Parameter } \\
\hline \multirow{2}{*}{$\begin{array}{l}\text { Tumor- } \\
\text { größe }\end{array}$} & pT1 & 44 & 14 & \multirow[t]{2}{*}{0,142} & 45 & 7 & \multirow[t]{2}{*}{0,396} \\
\hline & pT2-4 & 77 & 35 & & 79 & 16 & \\
\hline \multirow{2}{*}{$\begin{array}{l}\text { Lymphkno- } \\
\text { tenstatus }\end{array}$} & pNO & 55 & 11 & \multirow[t]{2}{*}{0,021} & 54 & 6 & \multirow[t]{2}{*}{0,033} \\
\hline & $\mathrm{pN1} 1-3$ & 59 & 24 & & 59 & 15 & \\
\hline \multirow{3}{*}{$\begin{array}{l}\text { Histolo- } \\
\text { gisches } \\
\text { Grading }\end{array}$} & G1 & 11 & 3 & \multirow[t]{3}{*}{0,018} & 10 & 1 & \multirow[t]{3}{*}{0,019} \\
\hline & G2 & 60 & 15 & & 58 & 7 & \\
\hline & G3 & 60 & 30 & & 54 & 17 & \\
\hline \multirow{3}{*}{$\begin{array}{l}\text { Histolo- } \\
\text { gischer } \\
\text { Tumortyp }\end{array}$} & Invasiv duktal & 107 & 33 & \multirow[t]{3}{*}{0,006} & 106 & 23 & \multirow[t]{3}{*}{0,877} \\
\hline & Invasiv lobulär & 18 & 9 & & 18 & 2 & \\
\hline & Andere & 9 & 7 & & 9 & 2 & \\
\hline \multirow{2}{*}{$\begin{array}{l}\text { Östrogen- } \\
\text { rezeptor- } \\
\text { status }\end{array}$} & $\begin{array}{l}\text { Negativ } \\
\left(\operatorname{IRS}^{c}=0-2\right)\end{array}$ & 46 & 15 & \multirow[t]{2}{*}{0,701} & 45 & 12 & \multirow[t]{2}{*}{0,027} \\
\hline & $\begin{array}{l}\text { Positiv (IRS= } \\
3-12)\end{array}$ & 82 & 32 & & 82 & 14 & \\
\hline \multirow{2}{*}{$\begin{array}{l}\text { Progeste- } \\
\text { ronrezep- } \\
\text { torstatus }\end{array}$} & $\begin{array}{l}\text { Negativ } \\
(\mathrm{IRS}=0-2)\end{array}$ & 43 & 18 & \multirow[t]{2}{*}{0,240} & 43 & 11 & \multirow[t]{2}{*}{0,059} \\
\hline & $\begin{array}{l}\text { Positiv (IRS= } \\
3-12)\end{array}$ & 85 & 29 & & 84 & 15 & \\
\hline \multirow{2}{*}{$\begin{array}{l}\text { ID4-Promo- } \\
\text { tor-Methy- } \\
\text { lierung }\end{array}$} & Negativ & 39 & 8 & \multirow[t]{2}{*}{0,036} & 39 & 5 & \multirow[t]{2}{*}{0,169} \\
\hline & Positiv & 76 & 28 & & 75 & 16 & \\
\hline \multicolumn{8}{|c|}{ 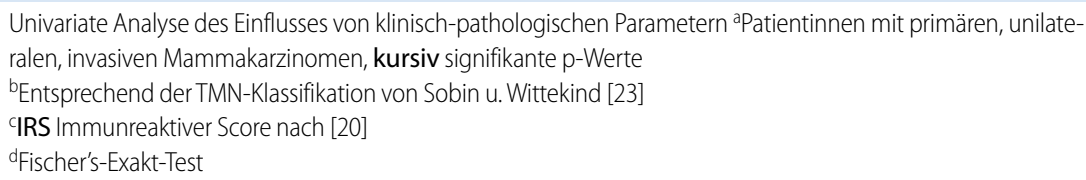 } \\
\hline
\end{tabular}

\section{Fazit für die Praxis}

Unsere Daten sprechen dafür, dass ID4 ein neues putatives Tumorsuppressorgen der humanen Brust darstellen könnte, welches während der Kanzerogenese durch aberrante Promotorhypermethylierung inaktiviert wird. Positive ID4Promotor-Methylierung stellt ferner einen möglichen prognostischen Marker für eine frühe Rezidivbildung des Mammakarzinoms dar und könnte somit als neuer Biomarker in der Behandlung von Brustkrebspatientinnen Verwendung finden. Unsere Untersuchungen bilden eine solide Basis für weiterführende funktionelle Analysen, mit dem Ziel, die Rolle von ID4 für die Progression und Metastasierung des humanen Mammakarzinoms weiter aufzuklären. Die Inaktivierung von Tumorsuppressorgenen durch aberrante Promotormethylierung bietet neue Möglichkeiten für die Identifizierung von DNA-basierten Biomarkern für humane Krebserkrankungen. So könnten DNA-Methylierungsmarkerpanels zukünftig eine verbesserte Krebsfrüherkennung, Risikoabschätzung, Chemoprädiktion und Kontrolle der Rezidivbildung ermöglichen, z. B. nach erfolgtem Nachweis dieser Biomarker in Körperflüssigkeiten wie Blut oder in archivierten Gewebeproben. Schließlich können solche Biomarker, aufgrund der Reversibilität von Methylierungsprozessen, möglicherweise in naher Zukunft auch als neue Zielmoleküle für effiziente Therapien dienen $[10,30]$.

\section{Korrespondenzadresse PD Dr. E. Dahl}

Arbeitsgruppe Molekulare Onkologie, Institut für Pathologie, Universitätsklinikum Aachen Pauwelsstraße 30, 52074 Aachen edahl@ukaachen.de

Interessenkonflikt. Der korrespondierende Autor gibt an, dass kein Interessenkonflikt besteht.

\section{Literatur}

1. Asirvatham AJ, Schmidt MA, Chaudhary J (2006) Non-redundant inhibitor of differentiation (Id) gene expression and function in human prostate epithelial cells. Prostate 66: 921-935

2. Beger C, Pierce LN, Kruger M et al. (2001) Identification of $I d 4$ as a regulator of $B R C A 1$ expression by using a ribozyme-library-based inverse genomics approach. Proc Natl Acad Sci USA 98: 130135

3. Cameron EE, Bachman KE, Myohanen S et al. (1999) Synergy of demethylation and histone deacetylase inhibition in the re-expression of genes silenced in cancer. Nat Genet 21: 103-107

4. Chan AS, Tsui WY, Chen X et al. (2003) Downregulation of ID4 by promoter hypermethylation in gastric adenocarcinoma. Oncogene 22: 6946-6953

5. Clark SJ, Harrison J, Paul CL et al. (1994) High sensitivity mapping of methylated cytosines. Nucleic Acids Res 22: 2990-2997

6. Dahl E, Kristiansen G, Gottlob K et al. (2006) Molecular profiling of laser-microdissected matched tumor and normal breast tissue identifies karyopherin alpha2 as a potential novel prognostic marker in breast cancer. Clin Cancer Res 12: 3950-3960

7. De Candia P, Akram M, Benezra R et al. (2006) Id4 messenger RNA and estrogen receptor expression: inverse correlation in human normal breast epithelium and carcinoma. Hum Pathol 37: 10321041

8. Desprez PY, Sumida T, Coppe JP (2003) Helix-loophelix proteins in mammary gland development and breast cancer. J Mammary Gland Biol Neoplasia 8: 225-239

9. Esteller M (2002) CpG island hypermethylation and tumor suppressor genes: a booming present, a brighter future. Oncogene 21: 5427-5440

10. Esteller M, Herman JG (2002) Cancer as an epigenetic disease: DNA methylation and chromatin alterations in human tumours. J Pathol 196: 1-7

11. Fink L, Seeger W, Ermert L et al. (1998) Real-time quantitative RT-PCR after laser-assisted cell picking. Nat Med 4: 1329-1333

12. Herman JG, Graff JR, Myohanen S et al. (1996) Methylation-specific PCR: a novel PCR assay for methylation status of $\mathrm{CpG}$ islands. Proc Natl Acad Sci USA 93: 9821-9826

13. Itahana Y, Singh J, Sumida T et al. (2003) Role of Id2 in the maintenance of a differentiated and noninvasive phenotype in breast cancer cells. Cancer Res 63: 7098-7105

14. Jones PA, Baylin SB (2007) The epigenomics of cancer. Cell 128: 683-692

15. Kleeff J, Ishiwata T, Friess H et al. (1998) The helixloop-helix protein Id 2 is overexpressed in human pancreatic cancer. Cancer Res 58: 3769-3772

16. Lin CQ, Singh J, Murata K et al. (2000) A role for Id1 in the aggressive phenotype and steroid hormone response of human breast cancer cells. Cancer Res 60: 1332-1340

17. Noetzel E, Veeck J, Niederacher D et al. (2008) Promoter methylation-associated loss of ID4 expression is a marker of tumour recurrence in human breast cancer. BMC Cancer 8: 154

18. Norton JD (2000) ID helix-loop-helix proteins in cell growth, differentiation and tumorigenesis. J Cell Sci 113: 3897-3905

19. Perk J, Gil-Bazo I, Chin Y et al. (2006) Reassessment of id1 protein expression in human mammary, prostate, and bladder cancers using a monospecific rabbit monoclonal anti-id1 antibody. Cancer Res 66: 10870-10877 
20. Remmele W, Stegner HE (1987) Recommendation for uniform definition of an immunoreactive score (IRS) for immunohistochemical estrogen receptor detection (ER-ICA) in breast cancer tissue. Pathologe 8 : 138-140

21. Roldan G, Delgado L, Muse IM (2006) Tumoral expression of BRCA1, estrogen receptor alpha and ID4 protein in patients with sporadic breast cancer. Cancer Biol Ther 5: 505-510

22. Shan L, Yu M, Qiu C et al. (2003) Id4 regulates mammary epithelial cell growth and differentiation and is overexpressed in rat mammary gland carcinomas. Am J Pathol 163: 2495-2502

23. Sobin LH, Wittekind C (1997) TNM classification of malignant tumours. Wiley, New York

24. Turner NC, Reis-Filho JS, Russell AM et al. (2007) BRCA1 dysfunction in sporadic basal-like breast cancer. Oncogene 26: 2126-2132

25. Umetani N, Takeuchi H, Fujimoto A et al. (2004) Epigenetic inactivation of ID4 in colorectal carcinomas correlates with poor differentiation and unfavorable prognosis. Clin Cancer Res 10: 74757483

26. Umetani N, Mori T, Koyanagi K et al. (2005) Aberrant hypermethylation of ID4 gene promoter region increases risk of lymph node metastasis in $\mathrm{T} 1$ breast cancer. Oncogene 24: 4721-4727

27. Veeck J, Niederacher D, An H et al. (2006) Aberrant methylation of the Wnt antagonist SFRP1 in breast cancer is associated with unfavourable prognosis. Oncogene 25: 3479-3488

28. Welcsh PL, Lee MK, Gonzalez-Hernandez RM et al. (2002) BRCA1 transcriptionally regulates genes involved in breast tumorigenesis. Proc Natl Acad Sci USA 99: 7560-7565

29. Wilson JW, Deed RW, Inoue T et al. (2001) Expression of Id helix-loop-helix proteins in colorectal adenocarcinoma correlates with p53 expression and mitotic index. Cancer Res 61: 8803-8810

30. Yang X, Yan L, Davidson NE (2001) DNA methylation in breast cancer. Endocr Relat Cancer 8: 115127

31. Yokota Y, Mori S (2002) Role of Id family proteins in growth control. J Cell Physiol 190: 21-28

32. Yu L, Liu C, Vandeusen J et al. (2005) Global assessment of promoter methylation in a mouse model of cancer identifies ID4 as a putative tumor-suppressor gene in human leukemia. Nat Genet 37: 265-274

33. Zebedee Z, Hara E (2001) Id proteins in cell cycle control and cellular senescence. Oncogene 20: 8317-8325 